\title{
AUTOMATIC DETECTION OF MOVING WILD ANIMALS IN AIRBORNE REMOTE SENSING IMAGES
}

\author{
Yu Oishi ${ }^{1}$ and Tsuneo Matsunaga ${ }^{2}$ \\ ${ }^{1}$ University of Tsukuba \\ ${ }^{2}$ National Institute for Environmental Studies
}

\section{INTRODUCTION}

The coexistence of wild animals with people needs proper conservation and management measures of wild animals including population control. However, there is not enough necessary information for management of large-sized mammals such as population sizes, habitat, etc [1]. This is partly because many of the mammals are nocturnal animals, and it is difficult to observe the large-sized mammals because of their large habitat area [2]. Therefore in Japan population densities of large mammals have been estimated using direct and indirect methods, although these methods are labor-intensive and require long-term research. Thus, it is expected to estimate population densities of large mammals using remote sensing.

In this study, we developed an algorithm for automatic detection of moving wild animals in the snow in airborne remote sensing images with $60 \%$ overlap taken in Sarufutsu Village, Hokkaido in Japan [3][4]. This is the first report on automatic detection of wild animals from remote sensing images except infrared thermal imagery in Japan.

\section{DATA}

The study area is Sarufutsu Village, northern Hokkaido in Japan. We used airborne remote sensing images

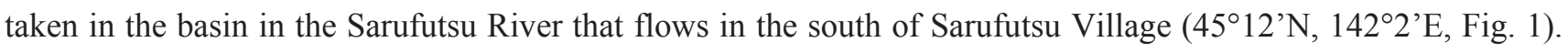
Fig. 2 shows birds-eye view of the taken area from the Okhotsk Sea generated from ASTER 3A01 product.

A geographical feature of this area could be as follow: $100 \pm 60 \mathrm{~m}$ above the sea, low difference of elevation, many meandering flows of river, and an artificial forest.

The 68 airborne remote sensing images used in this study were collected May 3, 2006 at an altitude of $750 \mathrm{~m}$ with $60 \%$ image overlap at an interval of 3 seconds. The pixel resolution of the imagery is about $8 \mathrm{~cm}$. The image size is about $600 \mathrm{~m}$ by $1 \mathrm{~km}$. And these images have four bands in the visible - near infrared region.

The target species in this study are brown bear, sika deer, fox, and human which can be recognized in $8 \mathrm{~cm}$ resolution imagery. 


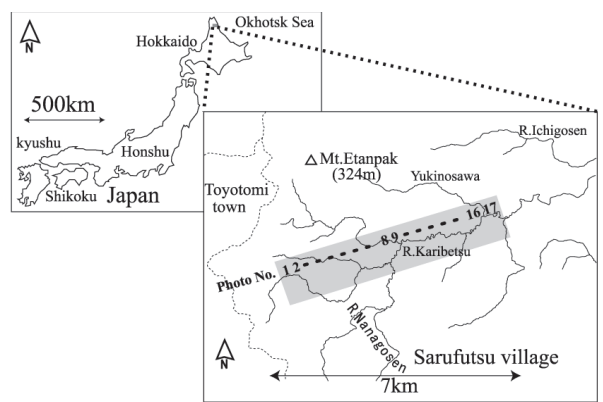

Fig. 1. The study area in Sarufutsu Village, Hokkaido, Japan (a gray rectangle is the area of aerial photographs).

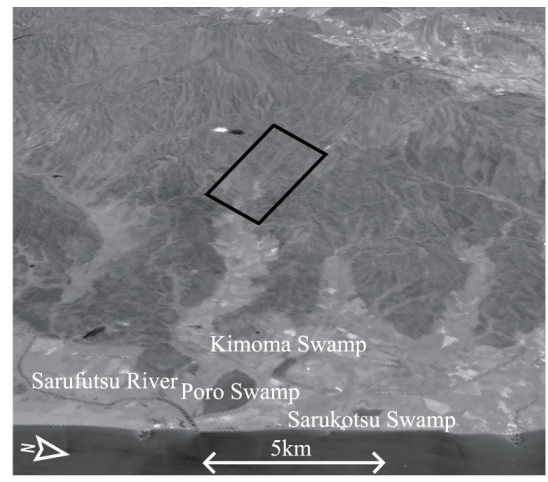

Fig. 2. Bird-eye view from the Okhotsk Sea generated from ASTER 3A01 product (a black rectangle is the area of aerial photographs) .

\section{MEHOTDS}

Fig. 3 shows the flowchart of the developed algorithm to detect moving animals in overlapping airborne remote sensing images.

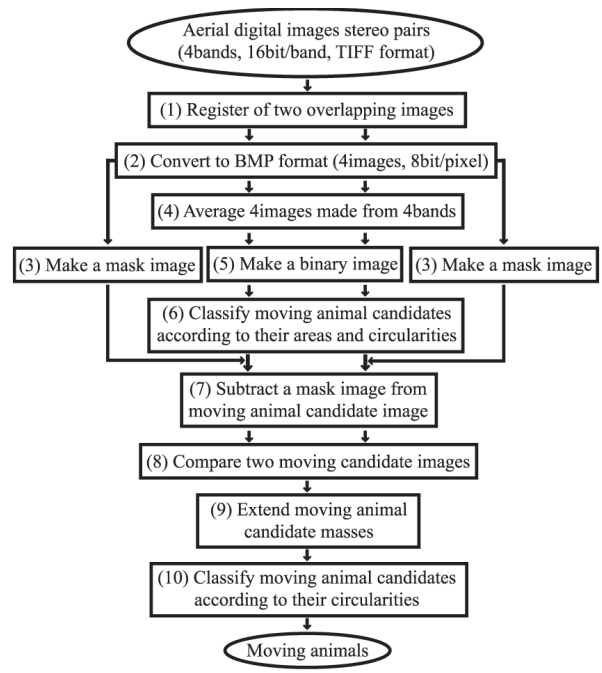

Fig. 3. The flowchart of the developed algorithm to detect the moving animals in overlapping airborne remote sensing images. 
1) Register of two overlapping images with Triangulation method of ENVI 4.4.

2) Convert to BMP format by converting minimum $D N$ value to 0 and maximum $D N$ value to 255 and linear interpolate between them.

3) Make a mask image of trees and rivers with NDVI (the normalized difference vegetation index).

4) Average four images made from four bands.

5) Make a binary image using a method incorporated with Laplacian-histogram method by adding moving window function, P-tile method, and Otsu method.

6) Classify moving animal candidates according to their areas and circularities. The area is determined from size of target animals and circularity is determined from circularity of the form that may be thought moving animals by visual observation.

7) Subtract a mask image from moving animal candidate image.

8) Compare two moving animal candidate images. Step 1), the tips of a tree taken in an image is dissimilar to them of a same tree taken in another image in a position and a form by tilt displacements effect. However, at the root of a tree the position and the form coincide. Therefore in case a part of a mass is superimposed between each images, we rejected this mass. Step 2), we compared the animal candidate mass and those within the range of a possible moving range in another image. And in case two compared masses resemble closely each other, we extracted the masses as moving animal candidates.

9) Extend moving animal candidate masses. The contours of moving animal masses are distinct. However, the contours of shadows of branches that cause many "dummy masses" (false detection of moving animals) are obscure. Therefore when surrounding DN value is closer to DN value of masses, we extended the masses.

10) Classify moving animal candidates according to their circularities. The extended dummy masses have low circularity.

\section{RESULTS}

Since we found a walking human in images used in this study, developed algorithm was applied to two images of 2000x2000 pixels including a human. Fig. 4 shows input images, human images taken from input images, and the human extracted in a result image. 


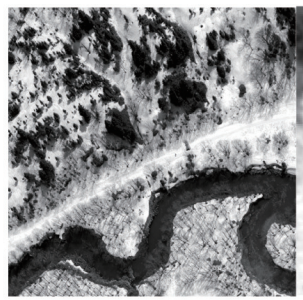

(a)

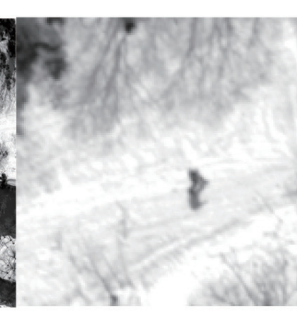

(b)

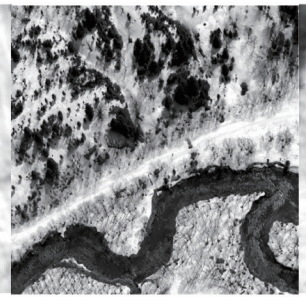

(c)

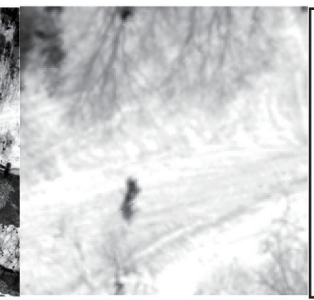

(d)

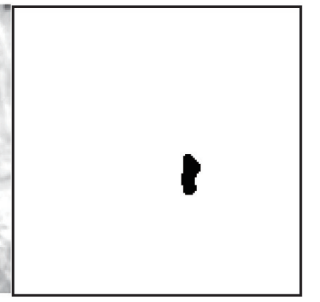

(e)

Fig. 4. Input images and a result image. (a) Input image 1. (b) A human in input image 1. (c) Input image2. (d) A human in input image 2. (e) A result image with an extracted human.

Our developed algorithm could extract the walking human from a total of 2192 masses. However 2 dummy masses are extracted as moving animal candidates.

\section{CONCLUSION}

Our developed algorithm is effective in detecting automatically the walking human in remote sensing images. And the developed algorithm that extracted 2 dummy masses in 2000x2000 pixels is validity as aerial image interpretation support system of moving wild animals. We will apply the developed algorithm to all the images to detect moving animals. And we will research the applications of detected moving animals for biological information such as population sizes, habitat, etc.

\section{REFERENCES}

[1]. T. Doi, T. Iwamoto, S. Miura, and H. Ikeda, “Ecology of Mammals”, University of Tokyo Press, Tokyo, 1997, p.261 (in Japanese).

[2]. S. Takatsuki, J. Yamagiwa et al., "Mammalogy in Japan”, University of Tokyo Press, Tokyo, 2008, p.472 (in Japanese).

[3]. Y. Oishi and T. Matsunaga, "Automatic detection of the moving wild animals in the snow in multi-temporal airborne remote sensing images", Proceedings of the 47th Autumn Conference of the Remote Sensing Society of Japan, Nagoya, Japan, Nov. 2009 (in Japanese).

[4]. Y. Oishi, T. Matsunaga, and O. Nakasugi, "Automatic detection of the tracks of wild animals in the snow in airborne remote sensing images and its use", submitted to Journal of the Remote Sensing Society of Japan, 2009 (in Japanese).

\section{ACKNOWLEDGEMENT}

The authors would like to thank M. Fukushima, T. Kameyama, and H. Shimazaki for providing airborne images and for their helpful comments, H. Yamano, H. Oguma for their helpful comments, and for all other help and cooperation of the people who live in Sarufutsu Village. 